\title{
A density functional theory study of poly(vinylidene difluoride) crystalline phases
}

\author{
Francesco Pelizza and Karen Johnston \\ Department of Chemical and Process Engineering, University of \\ Strathclyde, 75 Montrose Street, Glasgow G1 1XJ, United Kingdom
}

June 27, 2019

\begin{abstract}
The structure and energetics of crystalline phases of poly(vinylidene difluoride) were investigated using density functional theory (DFT). In particular, we present data on non-polar analogues of the $\beta$ and $\gamma$ phases that have not yet been experimentally characterised. The results from a variety of exchange and correlation functionals were compared and it was found that van der Waals (vdW) interactions have an important effect on the calculated crystal structures. While the relative phase energies were not strongly dependent on the functional, the cohesive energies were significantly affected.
\end{abstract}

\section{Introduction}

Ferroelectric (FE) materials, particularly thin films are widely used in electronic devices as non-volatile memory, capacitors and sensors [1]. The replacement of FE ceramics with FE polymers is desirable as polymers are compatible with a wide range of substrates, are easier to process and have mechanical flexibility. Poly(vinylidene difluoride) (PVDF) is the most promising FE polymer due to its chemical stability, low coercive field, large remnant polarisation and high Curie temperature $[2,3]$.

PVDF has six main crystal phases and native PVDF samples are typically composed of a mixture of these crystalline phases within amorphous regions. The PVDF crystalline phases, have three different chain structures with parallel or antiparallel 
chain orientations. If the chains are aligned to give a net dipole moment, then the crystal is polar, and since the direction of the dipole moment can be switched by an electric field the crystal is FE. If the chain dipoles are antiparallel then the crystal is non-polar and, therefore, non-FE. Figure 1 shows the all-trans (T) backbone, the trans-gauche $\mathrm{TG}^{+} \mathrm{TG}^{-}$backbone and the more complex $\mathrm{T}_{3} \mathrm{G}^{+} \mathrm{T}_{3} \mathrm{G}^{-}$backbone.

Figure 1: Structures of (a) all-trans all- $\mathrm{T}$ chain (4 repeat units), (b) $\mathrm{TG}^{+} \mathrm{TG}^{-}$chain (2 repeat units), )c) $\mathrm{T}_{3} \mathrm{G}^{+} \mathrm{T}_{3} \mathrm{G}^{-}$chain (1 repeat unit). The black arrow shows the dipole orientation perpendicular to the chain axis.

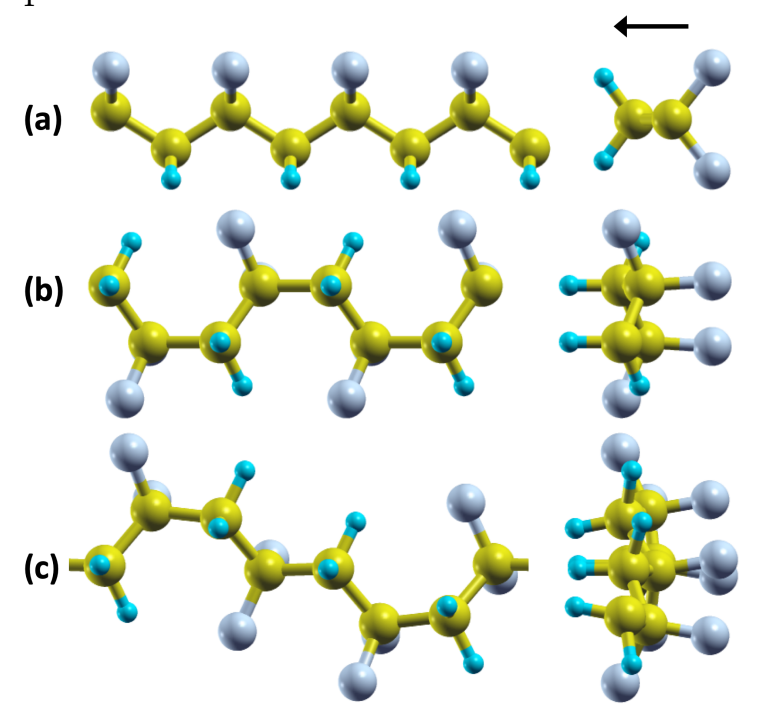

The $\beta$ phase is the most desirable PVDF phase, as it has the highest spontaneous polarisation of any FE polymer. There is a large body of experimental research focused on finding the optimal processing procedures for the formation of the $\beta$ phase. However, since multiple phases of PVDF are often present experimentally, accurate sample characterisation will depend on knowing the properties of all crystal phases. DFT is known to give a good prediction of the lattice parameters and structure of the $\alpha, \delta, \beta$ and $\gamma$ phases, but only a few DFT studies have investigated the $\mathrm{T}_{3} \mathrm{G}^{+} \mathrm{T}_{3} \mathrm{G}^{-}$ non-polar $\epsilon$ phases. However, the DFT studies for the $\epsilon$ phases did not account for van der Waals (vdW) interactions, which is known to be important for molecular crystals, including polymers $[4,5]$. In addition, there are no DFT studies of the nonpolar analogue of the $\beta$ phase, denoted here by $\zeta$, and it has not been characterised experimentally. 
In this paper we present the first DFT study of the properties of the non-polar PVDF $\zeta$ phase, and investigate the $\beta$ to $\zeta$ transition. We also present the first study of the $\epsilon$ phase using DFT that includes van der Waals (vdW) interactions. In addition, we calculate the $\gamma$ phases and compare our results for lattice structures, polarisation, relative phase energies, and cohesive energies to previous work on PVDF crystal phases.

\section{Method}

All calculations were performed using the Quantum Espresso DFT software package (QE versions 5.3 and 5.4) [6] that is implemented using a planewave basis set. The adopted threshold values were $80 \mathrm{Ry}$ for the wavefunction and $800 \mathrm{Ry}$ for the charge density. Several exchange and correlation (XC) functionals were used, including the local density approximation (LDA), the PBE generalised gradient approximation (GGA) [7], the van der Waals (vdW) functionals vdW-DF [8] and vdW-DF2 [9] and the semi-empirical vdW correction scheme DFT-D2 [10]. The Brillouin zone was sampled using a Monkhorst-Pack mesh of $2 \times 3 \times 4$ for the $\zeta$ phase, and $3 \times 1 \times 2$ for the $\gamma$ and $\epsilon$ phases. For core electrons we used ultrasoft pseudopotentials available on the QE website [11]. For the PBE, vdW-DF, vdW-DF2 and DFT-D2 XC functionals we used F.pbe-n-rrkjus_Psl.0.1.UPF, C.pbe-n-rrkjus.UPF and H.pbe-n-rrkjus.UPF, and for the LDA functional we used F.pz-van_asa.UPF, C.pz-van_ak.UPF and H.pzvan_ak.UPF.

For structural relaxations the thresholds were set to be $10^{-3}$ a.u. (atomic units) for the atomic forces and $10^{-4}$ Ry for the total energy. The self-consistency convergence threshold was $10^{-8} \mathrm{Ry}$. The $\zeta$ phase characterisation was performed with both force and total energy thresholds increased up to one order of magnitude in respect to what was used for the $\gamma$ and $\epsilon$ phases.

Space groups were determined using the FINDSYM code [12]. The spontaneous polarisation was calculated using the Berry phase method implemented in Quantum Espresso. The Berry phase calculations used $11 k$-points with 120 bands along the direction of the polarisation. 


\section{Results and Discussion}

\subsection{Structure of the $\zeta$-phase}

The main difference between the $\beta$ and $\zeta$ phases, is that in the $\beta$ phase the chain dipole moments are parallel, whereas the $\zeta$ phase the chain dipole moments are antiparallel resulting in a non-polar phase. The initial structure for the $\zeta$ phase was obtained from the $\beta$ phase by reflecting one of the chains in the $y$-direction (defined as the direction of the chain dipole moment) and relaxing the system. Upon relaxation of the $\zeta$ phase the two chains become coplanar in the $x y$ plane, as shown in Figure 2. The $\zeta$ phase has orthorhombic spacegroup Pmma (number 51) and the lattice parameters for the $\zeta$-phase are presented in Table 1. Experimental characterisation for the $\zeta$ phase is not available.

Figure 2: Unit cell of the PVDF $\zeta$-phase, showing that the chains have become coplanar in the $x y$-plane.

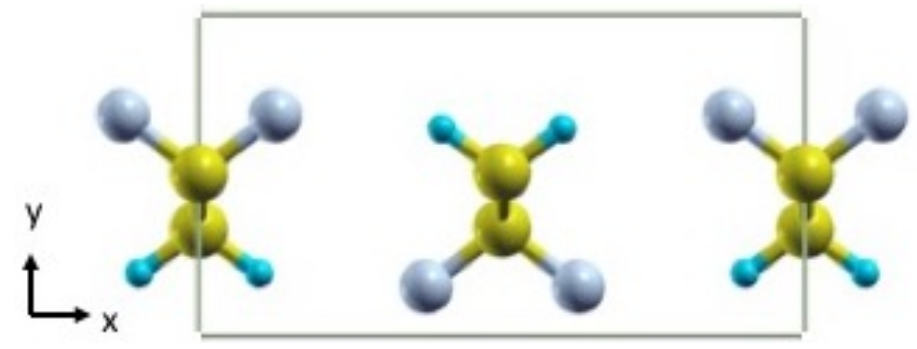

Despite the structural differences between the $\beta$ and $\zeta$ phases, the $\zeta$ lattice parameters are very similar to the $\beta$-phase lattice parameters [5]. As observed in this previous study of PVDF, the XC functional has a significant effect, with LDA predicting the smallest volume and PBE predicting the largest volume.

The only other simulation study that considered a non-polar all-trans phase was the DFT PBEsol [13] phase transition study by Kim et al [14]. Their structure, denoted $\beta\left(I_{a}\right)$, was proposed as a transition state from the non-polar $\alpha$ phase to the polar $\beta$ phase. However, this transition state differs from our proposed $\zeta$ structure. In their $\beta\left(I_{a}\right)$ phase the chains were not aligned but were positioned similar to the $\beta$ phase. They did note that this structure was unstable, and perhaps due to constraints imposed by the NEB method, they did not observe a relaxation to a coplanar structure. 
Table 1: Lattice constants for the $\zeta$ and $\beta$ phases using different XC functionals.

\begin{tabular}{rrrrl}
$a(\AA)$ & $b(\AA)$ & $c(\AA)$ & $\mathrm{V}\left(\AA^{3}\right)$ & Method \\
\hline \multicolumn{5}{c}{$\zeta$-phase } \\
7.96 & 4.45 & 2.54 & 89.9 & LDA \\
8.99 & 5.00 & 2.59 & 116.2 & PBE \\
8.65 & 4.77 & 2.60 & 107.1 & vdW-DF \\
8.42 & 4.64 & 2.60 & 101.5 & vdW-DF2 \\
8.26 & 4.56 & 2.58 & \multicolumn{5}{c}{$\beta 7.0$} & DFT-D2 \\
\hline \multicolumn{5}{c}{$\beta$-phase } \\
7.97 & 4.46 & 2.54 & 90.2 & LDA [5] \\
8.95 & 5.00 & 2.59 & 115.7 & PBE [5] $[5]$ \\
8.62 & 4.80 & 2.60 & 107.5 & vdW-DF [5 \\
8.40 & 4.66 & 2.60 & 101.8 & vdW-DF2 [5] \\
8.27 & 4.55 & 2.58 & 97.0 & DFT-D2 [5] \\
\hline
\end{tabular}

\subsection{Transition energy barrier from $\beta$ to $\zeta$ phase}

The transition routes and energy barrier between different phases of PVDF, particularly between the non-polar $\alpha$-phase and the polar $\beta$-phase, affect the formation of the $\beta$-phase from the $\alpha$-phase by stretching or poling and are, therefore, important in memory or energy storage device applications. Several previous studies have calculated the $\beta$ to $\alpha$-phase transition via different routes and phases. A study by Wang et al using the B3PW91 functional investigated the $\alpha$ to $\beta$ internal rotation barrier for short two-monomer chains and found a barrier of $16.3 \mathrm{~kJ} / \mathrm{mol}$ [15]. Another study by Ranjan et al investigated a transition route via the $\gamma$-phase $(\alpha$ to $\gamma$ to $\beta)$ using PBE and found the barriers to be 9.6 and $19.3 \mathrm{~kJ} / \mathrm{mol}(100$ and $50 \mathrm{meV})$, respectively [16]. A more recent study by Kim et al, using PBEsol functional, considered two different routes (Route I: $\alpha$ to $\delta$ to $\beta$, and Route II: $\alpha$ to a non-polar $\beta$-phase to $\beta$ ) and found that the barriers were and 3.4 and $6.8 \mathrm{~kJ} / \mathrm{mol}(53$ and $70 \mathrm{meV}$ ), respectively [14].

We investigated the energy barrier between the $\beta$ and $\zeta$ phases using both PBE and vdW-DF exchange and correlation functionals. We first performed a rigid rotation and translation, starting from the $\beta$-phase conventional unit cell (with two chains in the unit cell), then rigidly rotating one of the chains in stages to $180^{\circ}$ around the $z$-axis, then translating one of the chains along the $y$-direction to reach the $\zeta$-phase, which is shown in Fig. 3. For each stage, we then allowed the ions to partially relax 
at each stage. All $\mathrm{H}$ and $\mathrm{F}$ atom coordinates were allowed to relax but the $\mathrm{C}$ atom coordinates in the rotated chain were fixed in $x$ and $y$, and in the vertical chain were fixed in $x$, which retained the angle between the chains. and performed an electronic self-consistent calculation. Finally, we allowed the cell to change at each stage, to account for the different $\beta$ and $\zeta$ cell parameters. The results are shown in Fig. 3.

Figure 3: Transition energy barrier $(\mathrm{kJ} / \mathrm{mol})$ from $\beta$-phase $\left(0^{\circ}\right)$ to $\zeta$-phase $\left(180^{\circ}\right)$ using rigid rotation and translation (triangles), ionic relaxations (circles), and ionic and cell relaxations (squares) for PBE (hollow symbols) and vdW-DF (solid symbols). The insets show how relaxation allows the vertical chain to translate towards a more coplanar structure, reducing the energy barrier at $70^{\circ}$ and the energy of the $\zeta$-phase.

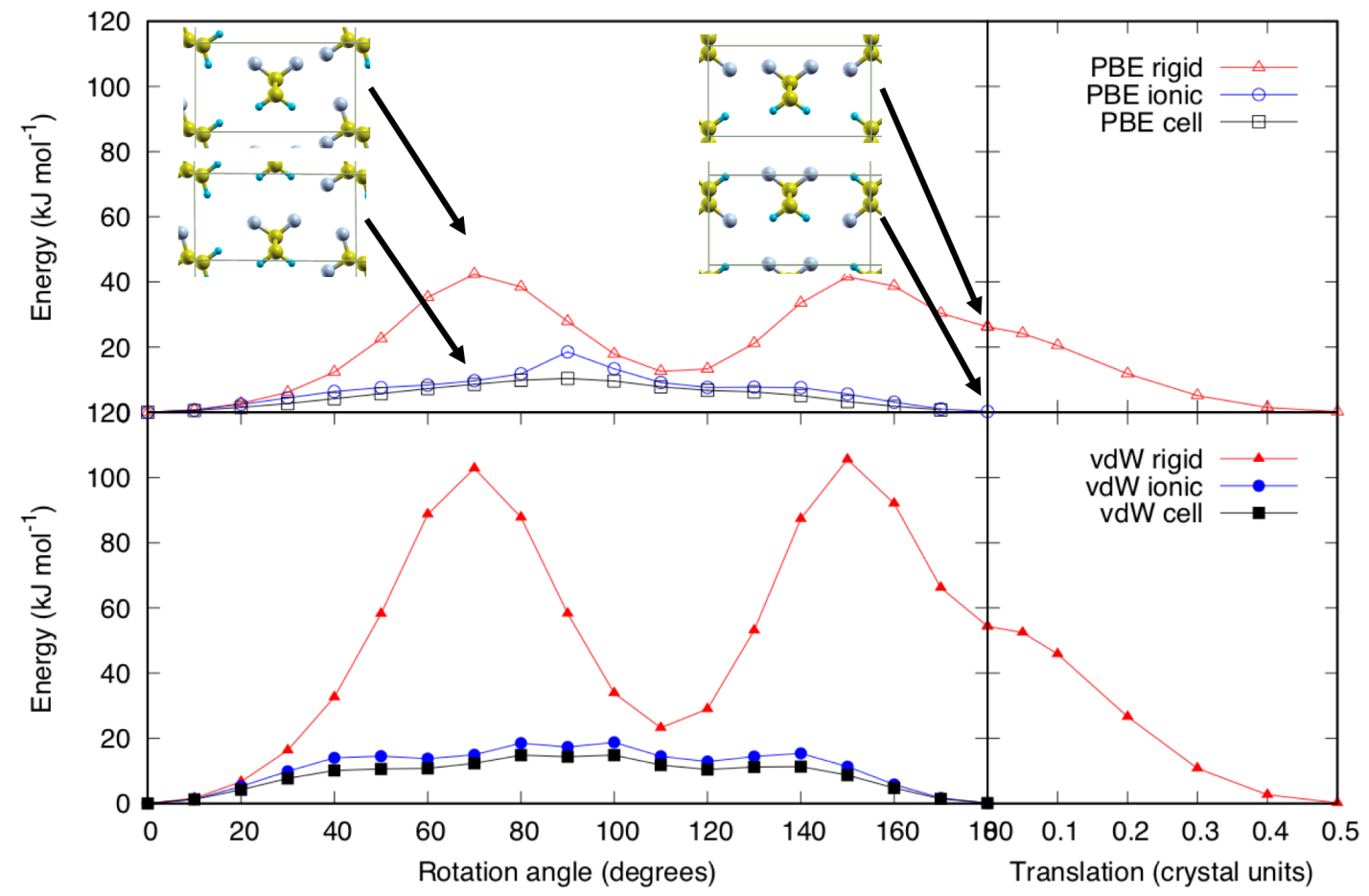

For both functionals, there are two maxima in the rigid transformation from $\beta$ to $\zeta$. For vdW-DF, the barriers for rigid rotation are $103 \mathrm{~kJ} / \mathrm{mol}$ and $106 \mathrm{~kJ} / \mathrm{mol}$ at $70^{\circ}$ and $150^{\circ}$, respectively. The energy of the unrelaxed non-polar phase is $54 \mathrm{~kJ} / \mathrm{mol}$ higher than the $\beta$-phase. There is no barrier for the rigid translation to the coplanar $\zeta$-phase. Relaxing the chains at each angle allows them to move towards the coplanar structure, 
reducing the energy barrier to $19 \mathrm{~kJ} / \mathrm{mol}$, as shown in the insets in Fig. 3. The energy of the relaxed non-polar $\zeta$-phase is approximately the same as the $\beta$-phase. Relaxing the cell volume results in only small reductions in the energy barrier to $15 \mathrm{~kJ} / \mathrm{mol}$. This value is comparable to the energy barriers calculated between other phases of PVDF discussed above. The PBE energies for the rigid transformation are much smaller than the vdW-DF energies although the maxima occur at the same rotation angle. However, the barriers for the relaxed transformations are not significantly different to the vdW-DF barriers with a maxima of $19 \mathrm{~kJ} / \mathrm{mol}$ and $10 \mathrm{~kJ} / \mathrm{mol}$ for ionic and cell relaxations, respectively.

\subsection{Structure of $\gamma$ - and $\epsilon$-phases}

The $\gamma$ and $\epsilon$ phases have a $\mathrm{T}_{3} \mathrm{G}^{+} \mathrm{T}_{3} \mathrm{G}^{-}$backbone structure, and are shown in Fig. 4 . The $\gamma$-phase has parallel chain dipole moments, making it FE, whereas the $\epsilon$-phase has antiparallel chain dipoles and is non-polar. There are two further variations in the crystal structures, so that the $\gamma$ and $\epsilon$ phases can each be represented by two very similar crystal structures. Fig. 4 shows how the two variants of the same PVDF crystal phase differ from each other by a $180^{\circ}$ rotation in the $x z$-plane of one of the chains with respect to the other. The distinction between these two variants within the same crystal phase is made from now on with the annotation uu (up-up) for parallel chains and ud (up-down) for anti-parallel chains. The $\gamma$ and $\epsilon$ phases are thus sub-distinguished as $\gamma_{\mathrm{uu}}, \gamma_{\mathrm{ud}}, \epsilon_{\mathrm{uu}}$ and $\epsilon_{\mathrm{ud}}$ as shown in Fig. 4.

We constructed the different crystal structures by applying symmetry operations to a $\mathrm{T}_{3} \mathrm{G}^{+} \mathrm{T}_{3} \mathrm{G}^{-}$chain in an orthorhombic cell. $\gamma_{\mathrm{uu}}$ was obtained by translation of the chain by $\left(\frac{1}{2}, \frac{1}{2}, 0\right)$ to give a structure with space group $C c$ (number 9 ). $\gamma_{\text {ud }}$ was obtained by rotating the chain around the $y$-axis followed by the above translation to get space group $P n a 2_{1}$ (number 33). To obtain the $\epsilon_{\text {uu }}$ structure, we rotated the chain by $180^{\circ}$ around the chain axis ( $z$-axis) followed by the above translation to give space group $P c a 2_{1}$ (number 29). Finally, to get $\epsilon_{\mathrm{ud}}$, the chain was rotated around the $z$-axis, then rotated around the $y$-axis, and finally translated to obtain a structure with space group $P 2_{1} / c$ (number 14 ). Our starting structures have the same space groups as those reported by Karasawa et al [17]. Pei et al [18] agreed with all $\mathrm{T}_{3} \mathrm{G}^{+} \mathrm{T}_{3} \mathrm{G}^{-}$crystal structures, except for $\epsilon_{\mathrm{ud}}$, where they reported a space group of $P 2_{c_{1}} / c$. We were unable to identify this space group and we surmise that this may be a typo, and should refer to $P 2_{1} / c$.

The lattice parameters for all $\gamma$ - and $\epsilon$-phases are shown in Table 2 . In all cases, the LDA functional gives the smallest lattice parameters and volumes and the PBE functional gives the largest lattice parameters for interchain directions and volumes. 
Figure 4: Assembly of crystal structures of PVDF phases $\gamma_{\mathrm{uu}}, \gamma_{\mathrm{ud}} \epsilon_{\mathrm{uu}}$ and $\epsilon_{\mathrm{ud}}$. Top panels show the view in the $x y$ plane where the dipole moment is in the $y$ direction and the bottom panels show along the chain in the $x z$ plane, highlighting the difference in up-up and up-down structures.
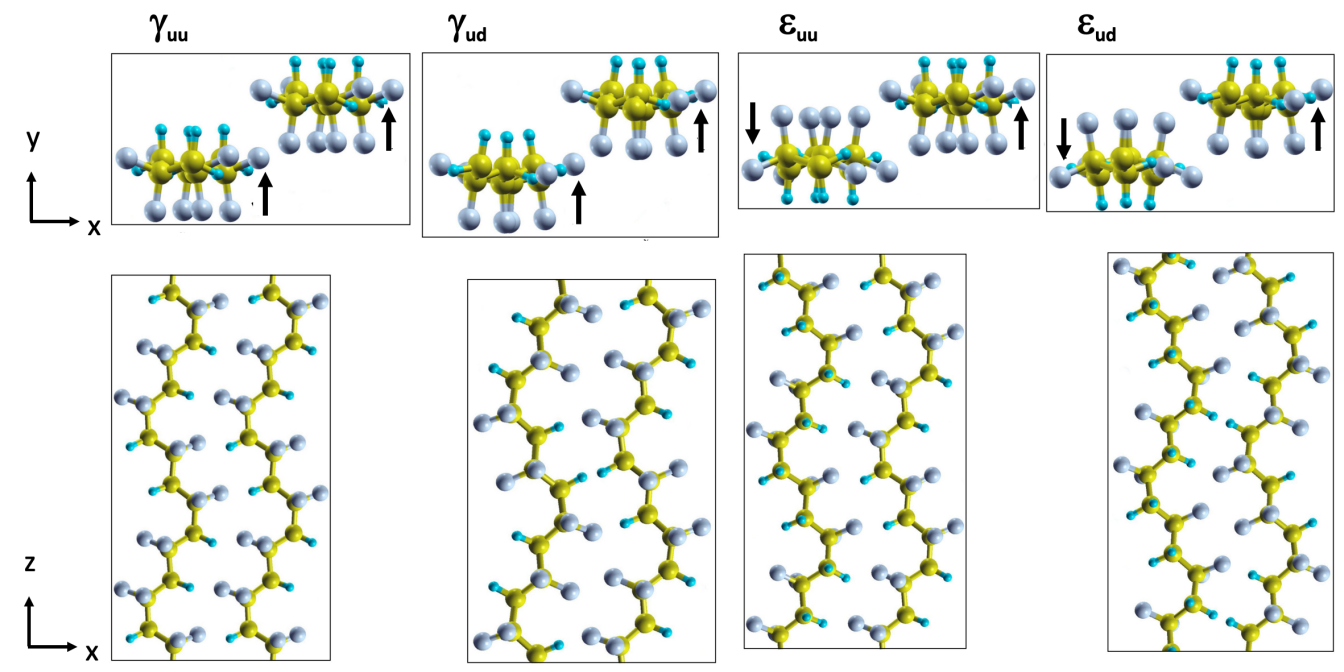

For all phases, our calculations using PBE give larger $a$ and $b$ lattice parameters, and a larger unit cell volume compared the PBE results of Pei et al [18]. We attribute this difference to methodological differences since Pei et al used a lower wavefunction cutoff ( $\approx 38-40 \mathrm{Ry})$ than this study $(80 \mathrm{Ry})$ and we note that a similar effect was previously observed for the $\alpha$ and $\delta$-phases [5]. The vdW-DF, vdW-DF2 and DFT-D2 functionals give lattice parameters in between those of the LDA and PBE functionals. This is the expected trend and is similar to the results for the $\zeta$-phase and also for previous results for the $\beta, \alpha$ and $\delta$-phases [5].

Experimentally, the $\gamma$-phase was first predicted to have an orthorhombic structure with space group $\mathrm{C} 2_{\mathrm{cm}}[19]$ and experimental samples contained both up-up (parallel) and up-down (antiparallel) chain packing. However, later studies showed that the structure is monoclinic [20,21]. The calculated structures of $\gamma_{\mathrm{uu}}$ and $\gamma_{\mathrm{ud}}$ phases both have monoclinic structures in agreement with experiment. The $\beta$ angle of the $\gamma_{u u}$ structures is in good agreement with experiment at a few degrees over $90^{\circ}$. The $\gamma_{\text {ud }}$ phase has the $\beta$ angle at around $90^{\circ}$ indicating that experimentalists observed $\gamma_{\mathrm{uu}}$.

The $\epsilon_{\mathrm{uu}}$ phase is orthorhombic with a negligible deviation of the $\beta$ angle from $90^{\circ}$ 
Table 2: Lattice parameters $a, b$ and $c$, unit cell volume, $V$, and angles $\alpha, \beta$ and $\gamma$ for the $\gamma_{\mathrm{uu}}, \gamma_{\mathrm{ud}}, \epsilon_{\mathrm{uu}}$ and $\epsilon_{\mathrm{ud}}$ phases.

\begin{tabular}{lrrrrrrrl}
\hline Phase & $a(\AA)$ & $b(\AA)$ & $c(\AA)$ & $\mathrm{V}\left(\AA^{3}\right)$ & $\alpha\left(^{\circ}\right)$ & $\beta\left(^{\circ}\right)$ & $\gamma\left(^{\circ}\right)$ & Method \\
\hline$\gamma$ & 9.66 & 4.97 & 9.18 & 440.7 & 90.0 & 90.0 & 90.0 & Expt. [19] \\
& 9.58 & 4.96 & 9.23 & 437.5 & 90.0 & 92.9 & 90.0 & Expt. [20] \\
& 9.67 & 4.96 & 9.20 & 440.1 & 90.0 & 93 & 90.0 & Expt. [21] \\
\hline$\gamma_{\text {uu }}$ & 9.09 & 4.59 & 9.09 & 379.1 & 90.0 & 93.0 & 90.0 & LDA \\
& 10.18 & 5.18 & 9.29 & 489.0 & 90.0 & 92.7 & 90.0 & PBE \\
& 9.71 & 4.95 & 9.34 & 447.7 & 90.0 & 94.4 & 90.0 & vdW-DF \\
& 9.41 & 4.85 & 9.33 & 424.8 & 90.0 & 94.5 & 90.0 & vdW-DF2 \\
& 9.38 & 4.78 & 9.24 & 414.2 & 90.0 & 93.3 & 90.0 & DFT-D2 \\
& 9.69 & 5.00 & 9.34 & 452.5 & 90.0 & 91.5 & 90.0 & PBE [18] \\
& 9.26 & 4.76 & 9.25 & 407.7 & 90.0 & 93.1 & 90.0 & DFT-D2 [18] \\
& 9.53 & 5.02 & 9.14 & 436.1 & 90.0 & 93.0 & 90.0 & MSXX [17] \\
\hline$\gamma_{\text {ud }}$ & 8.79 & 4.81 & 9.22 & 389.7 & 90.0 & 90.0 & 90.0 & LDA \\
& 10.78 & 5.29 & 9.39 & 534.6 & 90.0 & 91.7 & 90.0 & PBE \\
& 9.38 & 5.16 & 9.46 & 457.0 & 90.0 & 90.0 & 90.0 & vdW-DF \\
& 9.12 & 5.04 & 9.45 & 434.3 & 90.0 & 89.8 & 90.0 & vdW-DF2 \\
& 9.09 & 4.96 & 9.33 & 420.2 & 90.0 & 89.9 & 90.0 & DFT-D2 \\
& 9.75 & 5.17 & 9.39 & 463.5 & 90.0 & 90.0 & 90.0 & PBE [18] \\
& 9.27 & 5.24 & 9.28 & 450.8 & 90.0 & 90.0 & 90.0 & MSXX [17] \\
\hline$\epsilon_{\text {uu }}$ & 9.16 & 4.61 & 9.08 & 383.1 & 90.2 & 90.0 & 90.0 & LDA \\
& 10.09 & 5.18 & 9.30 & 486.2 & 90.1 & 90.0 & 90.0 & PBE \\
& 9.79 & 4.95 & 9.32 & 451.8 & 90.0 & 90.0 & 90.0 & vdW-DF \\
& 9.55 & 4.83 & 9.32 & 429.5 & 90.1 & 89.9 & 90.0 & vdW-DF2 \\
& 9.52 & 4.75 & 9.23 & 417.6 & 90.1 & 90.0 & 90.0 & DFT-D2 \\
& 9.78 & 5.08 & 9.33 & 463.5 & 90.0 & 90.0 & 90.0 & PBE [18] \\
& 9.68 & 4.98 & 9.13 & 440.1 & 90.0 & 90.0 & 90.0 & MSXX [17] \\
\hline & 8.87 & 4.84 & 9.17 & 393.8 & 90.0 & 93.0 & 90.1 & LDA \\
& 9.46 & 5.38 & 9.34 & 538.5 & 90.1 & 95.2 & 90.0 & PBE \\
& 9.18 & 5.07 & 9.41 & 438.0 & 90.0 & 93.3 & 90.0 & vdW-DF2 \\
& 9.21 & 4.95 & 9.30 & 424.2 & 90.2 & 91.2 & 90.1 & DFT-D2 \\
& 9.68 & 6.17 & 9.38 & 560.2 & 90.0 & 121.9 & 89.9 & PBE [18] \\
& 9.26 & 6.04 & 9.01 & & 90.0 & 120.0 & 90.0 & MSXX [17] \\
\hline & & & & & & & &
\end{tabular}


for some functionals, while the $\epsilon_{\mathrm{ud}}$ phase is monoclinic with negligible deviations of the $\alpha$ and $\gamma$ angles from $90^{\circ}$. There are no experimental measurements of the structure of $\epsilon$-phase, other than a report of its existence [22]. We know of only two studies of the $\epsilon$ phase, a DFT study by Pei et al [18] and a classical force field study by Karasawa and Goddard [17]. Although we agree with the space groups, we disagree with the crystal structure for $\epsilon_{\mathrm{ud}}$. Both the previous studies give an angle of $\beta \approx 120^{\circ}$, whereas our structure is close to orthorhombic with all angles $\approx 90^{\circ}$. We did attempt to create a structure with $\beta=120^{\circ}$ by modifying our $\epsilon_{\mathrm{ud}}$ structure, but we were unable to obtain a stable structure. However, we note that our results are in line with the structures of the other $\mathrm{T}_{3} \mathrm{G}^{+} \mathrm{T}_{3} \mathrm{G}^{-}$phases.

\subsection{Polarisation of the $\gamma$ phase}

The modern theory of the polarisation was used to determine the spontaneous polarisation of the $\gamma$ phases. To do this, it is necessary to have reference crystal structures to investigate. The first reference is a centrosymmetric crystal, therefore non-polar, and the second reference is a crystal with all atoms rotated by $180^{\circ}$ around the chain axis. In this study we have reported the polarisation in the direction perpendicular to the chains.

The results for the spontaneous polarisation of $\gamma_{\mathrm{uu}}$ and $\gamma_{\mathrm{ud}}$ phases are shown in Table 3. For both $\gamma_{\mathrm{uu}}$ and $\gamma_{\mathrm{ud}}$ phases the same spontaneous polarisation prediction trend of different functionals was observed. The highest spontaneous polarisation values were reached with the LDA functional. The vdW-DF and PBE XC functionals predicted the spontaneous polarisation being within the mid range of values reached within this characterisation. The vdW-DF2 and DFT-D2 functionals saw the spontaneous polarisation being towards the bottom range of predicted values.

\begin{tabular}{rrl}
\hline$\gamma_{u u}$ & $\gamma_{u d}$ & Method \\
\hline 13.2 & 16.0 & LDA \\
8.2 & 9.1 & PBE \\
8.4 & 10.7 & vdW-DF \\
6.9 & 5.8 & vdW-DF2 \\
7.8 & 7.0 & DFT-D2 \\
7.1 & - & PBE0 [23] \\
$0.2-0.3$ & Expt. [24] \\
\hline
\end{tabular}

Table 3: Spontaneous polarisation $\left(\mu \mathrm{C} \mathrm{cm}^{-2}\right)$ of $\gamma_{\mathrm{uu}}$ and $\gamma_{\mathrm{ud}}$ phases. 
Itoh et al [23] used the PBE0 XC functional and showed the spontaneous polarisation of the the $\gamma$ phase being $7.1 \mu \mathrm{C} \mathrm{cm}^{-2}$ for a $C c$ space group, which is in line with our results. An experimental study by Zhao et al on PVDF thin films found a very small polarisation of $0.2-0.3 \mu \mathrm{C} \mathrm{cm}^{-2}$ for the $\gamma$ phase (see Fig. $5 \mathrm{c}$ in their paper) [24]. It is typical that experimental measurements have a much smaller polarisation due to disorder in the sample.

\subsection{Phase and Cohesive Energies}

The total energy per monomer of the $\zeta, \gamma$ and $\epsilon$ PVDF phases relative to the stable $\alpha$ phase were calculated for different functionals, and are reported in Table 4 . The energetic ordering of the crystal phases depends on the $\mathrm{XC}$ functional. For example, LDA predicts that the $\gamma_{\mathrm{ud}}$ and $\epsilon_{\mathrm{ud}}$ are higher in energy than $\zeta$ but PBE predicts that $\zeta$ has the highest energy. All vdW functionals predict that $\gamma_{\mathrm{ud}}$ and $\epsilon_{\mathrm{ud}}$ are highest in energy, followed by $\zeta$, followed by $\epsilon_{\mathrm{uu}}$ and then $\gamma_{\mathrm{uu}}$. However, we note that the energy differences here are very small and are within the DFT error.

\begin{tabular}{rrrrrrl}
\hline$\beta$ & $\zeta$ & $\gamma_{\mathrm{uu}}$ & $\gamma_{\mathrm{ud}}$ & $\epsilon_{\mathrm{uu}}$ & $\epsilon_{\mathrm{ud}}$ & Method \\
\hline 5.1 & 4.9 & 3.5 & 10.3 & 4.6 & 10.1 & LDA \\
6.5 & 6.7 & 0.9 & 4.5 & 1.1 & 4.6 & PBE \\
3.8 & 3.9 & 0.2 & 5.9 & 0.9 & 6.0 & vdW-DF \\
2.6 & 2.7 & 0.2 & 7.2 & 1.2 & 7.2 & vdW-DF2 \\
3.5 & 3.3 & 0.7 & 5.5 & 1.2 & 5.3 & DFT-D2 \\
3.4 & 6.8 & - & - & - & - & PBEsol [14] \\
4.9 & - & 0.7 & - & 1.8 & - & PBE [25] \\
4.6 & - & 6.2 & - & - & - & PBE [16] \\
2.9 & - & 0.3 & - & - & - & PBE [23] \\
-3.6 & - & -2.3 & 4.4 & -1.6 & 1.5 & MSXX [17] \\
\hline
\end{tabular}

Table 4: PVDF phase energies in $\mathrm{kJ} / \mathrm{mol}$ per monomer relative to the $\alpha$ phase.

The $\zeta$ phase has not previously been calculated but we can compare its relative phase energy to a non-polar all-trans structure, calculated by Kim et al [14]. They obtained an relative phase energy of $70 \mathrm{meV}(6.75 \mathrm{~kJ} / \mathrm{mol})$ per monomer. While the phase energy is in good agreement with our PBE value, we note that their structure differs as it did not come from a structural relaxation, but rather from an intermediate transition state (image 5 in their paper) in a nudged elastic band calculation. The $\zeta$ phase energies are also very similar to previously reported $\beta$ phase energies for all functionals [5]. 
There are two studies that have calculated the phase energy of the $\gamma$ phase. Itoh et al calculated the phase energy per monomer for $\gamma_{\mathrm{uu}}$ to be $0.2 \mathrm{~kJ} / \mathrm{mol}$. Su et al calculated the relative phase energy for $\gamma_{\text {uu }}$ as $0.08 \mathrm{kcal} / \mathrm{mol}$ per carbon atom (equivalent to $0.67 \mathrm{~kJ} / \mathrm{mol}$ per monomer) and the relative phase energy for $\epsilon_{\mathrm{uu}}$ as $0.21 \mathrm{kcal} / \mathrm{mol}$ per carbon atom (equivalent to $1.76 \mathrm{~kJ} / \mathrm{mol}$ per monomer). Our PBE results are slightly higher for $\gamma_{\mathrm{uu}}$ and slightly lower for $\epsilon_{\mathrm{uu}}$, but again we note these energy differences are very small and overall our results are in good agreement with literature. Based on present and previously published results [5], all phase energies per monomer vary within a range of less than $10 \mathrm{~kJ} / \mathrm{mol}$ for all XC functionals. This narrow energy range at least partially explains why multiple phases are found within PVDF samples.

Cohesive energies were calculated to understand further the effect of vdW dispersion forces. The isolated, infinite chains were relaxed in a box with $a=b=19 \AA$ and with the $c$ lattice parameter (backbone direction) allowed to vary. The cohesive energy per monomer, $E_{\mathrm{coh}}$, of $\zeta, \gamma$ and $\epsilon$ phases are defined as:

$$
E_{\text {coh }}=\frac{E_{\text {crys }}-N_{\text {chain }} E_{\text {chain }}}{N_{\text {chain }} M_{\text {chain }}}
$$

where $E_{\text {crys }}$ is the lattice of the crystal, $E_{\text {chain }}$ is the total energy of one isolated chain in vacuum, $N_{\text {chain }}$ is the number of chains in the crystal unit cell, and $M_{\text {chain }}$ is the number of monomers in a chain.

The difference between the $\mathrm{T}_{3} \mathrm{G}^{+} \mathrm{T}_{3} \mathrm{G}^{-}$and all- $\mathrm{T}$ chain energies, $\Delta E_{\text {chain }}$, for the different functionals are shown in Table 5. $\Delta E_{\text {chain }}$ is similar for all functionals, although slightly lower for LDA and DFT-D2, which is expected since the differences depend on intrachain properties rather than interchain, so vdW would not play a strong role. These values are in line with previous calculation of -1.41 kcal per $\mathrm{C}$ atom $(-11.8 \mathrm{~kJ} / \mathrm{mol}$ per monomer) by $\mathrm{Su}$ et al [25].

The cohesive energies calculated using the various XC functionals are shown in Table 5. $E_{\text {coh }}$ were weakest for PBE, while vdW-DF and vdW-DF2 gave the strongest cohesive energies. LDA gave very similar results to DFT-D2. Our PBE results for $\gamma_{\text {uu }}$ and $\gamma_{\text {ud }}$ are similar to the PBE results calculated by $\mathrm{Su}$ et al and slightly lower than the PBE result of $-18.1 \mathrm{~kJ} / \mathrm{mol}$ for $\gamma_{\mathrm{uu}}$ by Pei et al [18].

The cohesive energies for the $\zeta$ phase are very similar to previous results for the $\beta$ phase [5] and these two all- $T$ phases have the strongest cohesive energies of all the phases. We observe that both $\mathrm{TG}^{+} \mathrm{TG}^{-}$structures reported previously have very similar energies. For the $\mathrm{T}_{3} \mathrm{G}^{+} \mathrm{T}_{3} \mathrm{G}^{-}$phases we note that the two up-up structures have similar energies, and the two up-down structures have similar cohesive energies, but that $\gamma_{\mathrm{ud}}$ and $\epsilon_{\mathrm{ud}}$ have higher cohesive energies than their $\gamma_{\mathrm{uu}}$ and $\epsilon_{\mathrm{uu}}$ counterparts. 
Table 5: Cohesive energies of the different phases in $\mathrm{kJ} / \mathrm{mol}$ per monomer. $\Delta E_{\text {chain }}$ energy $\left(\mathrm{kJ} / \mathrm{mol}\right.$ per monomer) is the energy of the $\mathrm{T}_{3} \mathrm{G}^{+} \mathrm{T}_{3} \mathrm{G}^{-}$chain relative to the all-T chain.

\begin{tabular}{rrrrrrl}
$\zeta$ & $\gamma_{\mathrm{uu}}$ & $\gamma_{\mathrm{ud}}$ & $\epsilon_{\mathrm{uu}}$ & $\epsilon_{\mathrm{ud}}$ & $\Delta E_{\text {chain }}$ & Method \\
\hline-41.8 & -29.8 & -23.1 & -28.7 & -23.2 & -13.4 & LDA \\
-17.4 & -10.5 & -6.9 & -10.3 & -6.8 & -12.6 & PBE \\
-47.4 & -38.5 & -32.8 & -37.8 & -32.7 & -12.6 & vdW-DF \\
-46.7 & -36.5 & -29.5 & -35.5 & -29.5 & -12.7 & vdW-DF2 \\
-41.7 & -30.2 & -25.4 & -29.7 & -25.6 & -14.2 & DFT-D2 \\
- & -10.0 & - & -8.6 & - & -11.8 & PBE [25] \\
- & -18.1 & - & - & - & - & PBE [18] \\
- & -59.8 & - & - & - & - & DFT-D2 [18] \\
\hline
\end{tabular}

Since the up-down phases have larger interchain lattice parameters, particularly the a lattice parameter, this results in weaker interchain interactions and hence lower cohesive energies.

\section{Conclusions}

The properties of several PVDF phases have been determined using DFT with a variety of $\mathrm{XC}$ functionals. The choice of functional significantly influenced the lattice parameters, particularly in the interchain crystal directions, and the cohesive energies. For the $\gamma$ phase the vdW functionals resulted in lattice parameters that agreed well with experimental values. In all phases studied, the PBE functional, which does not account for vdW interactions, was found to significantly overestimate the interchain lattice parameters.

We predicted the structure for the non-polar analogue of the all-trans $\beta$ phase, named here as the $\zeta$ phase, and found it to have a Pmma space group with the chains being coplanar in direction of the chain dipole moments. The $\beta$ to $\zeta$ transition energy barrier was calculated and was of the order of 10 and $19 \mathrm{~kJ} / \mathrm{mol}$ for vdW and PBE functionals, respectively, which is comparable to the energy barriers calculated for other PVDF phase transitions.

For the $\mathrm{T}_{3} \mathrm{G}^{+} \mathrm{T}_{3} \mathrm{G}^{-}$phases we determined the space groups of our structures, in agreement with previous literature. However, for $\epsilon_{\mathrm{ud}}$ we report a monoclinic angle just over $90^{\circ}$, compared to the $120^{\circ}$ angle predicted by previous studies $[17,18]$.

The energetic ordering of the phases was strongly dependent on the $\mathrm{XC}$ functional. 
PBE predicted that the $\zeta$ phase had a higher energy than all $\mathrm{T}_{3} \mathrm{G}^{+} \mathrm{T}_{3} \mathrm{G}^{-}$phases, whereas all other functionals found that $\gamma_{\mathrm{ud}}$ and its non-polar analogue $\epsilon_{\mathrm{ud}}$ had the highest energies. All functionals found that the up-down variants of the $\gamma$ and $\epsilon$ phases have higher energy than the up-up structures. This is mainly due to the low cohesive energy of these structures, which have larger interchain lattice parameters.

In conclusion, we investigated the experimentally uncharacterised $\epsilon$ and $\zeta$ phases using DFT. This provides information about the crystal structures that will aid future experimental characterisation, such as X-ray diffraction, of polycrystalline PVDF samples.

\section{Acknowledgements}

Results were obtained using the EPSRC funded ARCHIE-WeSt High Performance Computer (www.archie-west.ac.uk) EPSRC grant number EP/K000586/1. FP would like to thank Prof. Alan Kennedy for useful discussions on crystallography. Crystal structures for PVDF are available from http://dx.doi.org/10.15129/1108dcba-bc7542ef-a0ce-b95a89284e01.

\section{References}

[1] N. Setter, D. Damjanovic, L. Eng, G. Fox, S. Gevorgian, S. Hong, A. Kingon, H. Kohlstedt, N. Y. Park, G. B. Stephenson, I. Stolitchnov, A. K. Tagantsev, D. V. Taylor, T. Yamada, and S. Streiffer. Ferroelectric thin films: Review of materials, properties, and applications. J. Appl. Phys., 100:051606, 2006.

[2] Matthew Poulsen and Stephen Ducharme. Why Ferroelectric Polyvinylidene Fluoride is Special. IEEE Transactions on Dielectrics and Electrical Insulation, 17:1028-1035, 2010.

[3] R. Korlacki, J. Travis Johnston, Jihee Kim, Stephen Ducharme, Daniel W. Thompson, V. M. Fridkin, Zhongxin Ge, and James M. Takacs. Oligo(vinylidene fluoride) Langmuir-Blodgett films studied by spectroscopic ellipsometry and the density functional theory. J. Chem. Phys., 129:064704, 2008.

[4] J. Kleis, B. I. Lundqvist, D. C. Langreth, and E. Schröder. Towards a working density-functional theory for polymers: First-principles determination of the polyethylene crystal structure. Phys. Rev. B, 76:100201(R), 2007. 
[5] F. Pelizza, B.R. Smith, and K. Johnston. A van der waals density functional theory study of poly(vinylidene difluoride) crystalline phases. The European Physical Journal Special Topics, 225:1733, 2016.

[6] Paolo Giannozzi, Stefano Baroni, Nicola Bonini, Matteo Calandra, Roberto Car, Carlo Cavazzoni, Davide Ceresoli, Guido L. Chiarotti, Matteo Cococcioni, Ismaila Dabo, Andrea Dal Corso, Stefano de Gironcoli, Stefano Fabris, Guido Fratesi, Ralph Gebauer, Uwe Gerstmann, Christos Gougoussis, Anton Kokalj, Michele Lazzeri, Layla Martin-Samos, Nicola Marzari, Francesco Mauri, Riccardo Mazzarello, Stefano Paolini, Alfredo Pasquarello, Lorenzo Paulatto, Carlo Sbraccia, Sandro Scandolo, Gabriele Sclauzero, Ari P. Seitsonen, Alexander Smogunov, Paolo Umari, and Renata M. Wentzcovitch. QUANTUM ESPRESSO: a modular and open-source software project for quantum simulations of materials. J. Phys.: Condens. Matter, 21(39):395502, 2009.

[7] J.P. Perdew, Burke, Kieron, and Matthias Ernzerhof. Generalized gradient approximation made simple. Phys. Rev. Lett., 77:3865-3868, 1996.

[8] M. Dion, H. Rydberg, E. Schröder, D. C. Langreth, and B. I. Lundqvist. Van der waals density functional for general geometries. Phys. Rev. Lett., 92(24):246401, 2004 .

[9] Kyuho Lee, Eamonn D. Murray, Lingzhu Kong, Bengt I. Lundqvist, and David C. Langreth. Higher-accuracy van der Waals density functional. Phys. Rev. B, 82:081101(R), 2010.

[10] S. Grimme. Semiempirical GGA-type density functional constructed with a longrange dispersion correction. Journal of Computational Chemistry, 27:1787, 2006.

[11] http://www.quantum-espresso.org/pseudopotentials.

[12] H. T. Stokes and D. M. Hatch. FINDSYM: Program for Identifying the Space Group Symmetry of a Crystal. J. Appl. Cryst., 38:237, 2005.

[13] J. P. Perdew, A. Ruzsinzky, G. I. Csonka, O. A. Vydrov, G. E. Scuseria, L. A. Constantin, X. Zhou, and K. Burke. Restoring the Density-Gradient Expansion for Exchange in Solids and Surfaces. Phys. Rev. Lett., 100:136406, 2008.

[14] Won June Kim, Myung Hoon Han, Young-Han Shin, Hyungjun Kim, and Eok Kyun Lee. First-Principles Study of the $\alpha-\beta$ Phase Transition of Ferroelectric Poly(vinylidiene difluoride): Observation of Multiple Transition Pathways. J. Phys. Chem. B, 120(12):3240-3249, 2016. 
[15] Weijia Wang, Huiqing Fan, and Yangxue Ye. Effect of electric field on the structure and piezoelectric properties of poly(vinylidene fluoride) studied by density functional theory. Polymer, 51:3575-3581, 2010.

[16] V. Ranjan, Marco Buongiorno Nardelli, and J. Bernholc. Electric Field Induced Phase Transitions in Polymers: A Novel Mechanism for High Speed Energy Storage. Phys. Rev. Lett., 108:087802, 2012.

[17] Naoki Karasawa and William A. Goddard III. Force Fields, Structures and Properties of Poly(vinylidene fluoride) Crystals. Macromolecules, 25:7268, 1992.

[18] Y. Pei and X. C. Zeng. Elastic properties of poly(vinyldene fluoride) (PVDF) crystals: A density functional theory study. Journal of Applied Physics, 109:093514, 2011.

[19] S. Weinhold, M.H. Litt, and J.B. Lando. The crystal structure of the $\gamma$-phase of the poly(vinylidene fluoride). Macromolecules, 13:1178, 1980.

[20] Yasuhiro Takahashi, Masaki Kohyama, Yoshiaki Matsubara, Hisashi Iwane, and Hiroyuki Tadokoro. Tilting Phenomena in Forms II and III of Poly(vinylidene fluoride): Evidence for Monoclinic Structures. Macromolecules, 14:1841-1842, 1981.

[21] A.J. Lovinger. Unit cell of the $\gamma$ phase of poly(vinylidene fluoride). Macromolecules, 14(2):322-325, 1981.

[22] A. J. Lovinger. Annealing of Poly(vinylidene fluoride) and Formation of a Fifth Phase. Macromol., 15:40-44, 1982.

[23] Akira Itoh, Yoshiyuki Takahashi, Takeo Furukawa, and Hirofumi Yajima. Solid-state calculations of poly(vinylidene fluoride) using the hybrid DFT method:spontaneous polarization of polymorphs. Polymer Journal, 46:207-211, 2014.

[24] Yuetao Zhao, Wenyao Yang, Yujiu Zhou, Yan Chen, Xin Cao, Yajie Yang, Jianhua $\mathrm{Xu}$, and Yadong Jiang. Effect of crystalline phase on the dielectric and energy storage properties of poly(vinylidene fluoride). J. Mater Sci: Mater Electron, 27:7280-7286, 2016.

[25] Haibin Su, Alejandro Strachan, and William A. Goddard III. Density functional theory and molecular dynamics sturies of the energetics and kinetics of electroactive polymers: PVDF and P(VDF-TrFE). Phys. Rev. B, 70:064101, 2004. 